\title{
Efficient Computation of Adjusted $p$-Values for Resampling-Based Stepdown Multiple Testing*
}

\author{
Joseph P. Romano ${ }^{\dagger}$ \\ Departments of Statistics and Economics \\ Stanford University \\ Stanford, CA 94305, USA \\ romano@stanford.edu
}

\author{
Michael Wolf \\ Department of Economics \\ University of Zurich \\ 8032 Zurich, Switzerland \\ michael.wolf@econ.uzh.ch
}

February 2016

\begin{abstract}
There has been a recent interest in reporting $p$-values adjusted for resampling-based stepdown multiple testing procedures proposed in Romano and Wolf (2005a,b). The original papers only describe how to carry out multiple testing at a fixed significance level. Computing adjusted $p$-values instead in an efficient manner is not entirely trivial. Therefore, this paper fills an apparent gap by detailing such an algorithm.
\end{abstract}

KEY WORDS: Adjusted p-values; Multiple testing; Resampling; Stepdown procedure.

JEL classification code: C12.

*We thank Henning Müller for helpful comments.

${ }^{\dagger}$ Research supported by NSF Grant DMS-1307973. 


\section{Introduction}

Romano and Wolf $(2005 \mathrm{a}, \mathrm{b})$ propose resampling-based stepdown multiple testing procedures to control the familywise error rate (FWE); also see Romano et al. (2008, Section 3). The procedures as described are designed to be carried out at a fixed significance level $\alpha$. Therefore, the result of applying such a procedure to a set of data will be a 'list' of binary decisions concerning the individual null hypotheses under study: reject or do not reject a given null hypothesis at the chosen significance level $\alpha$.

In a series of recent papers, however, there has been an interest in computing adjusted $p$-values instead. ${ }^{1}$ That is, for each null hypothesis under study, compute a corresponding $p$-value adjusted for stepdown multiple testing proposed in Romano and Wolf (2005a,b). Examples of such papers include Heckman et al. (2010), Hein et al. (2010), Campbell et al. (2014), Gertler et al. (2014), and Dobbie and Fryer (2015). Unfortunately, the descriptions in these papers of how to compute the adjusted $p$-values are often unclear or even missing altogether.

In principle, for a given individual hypothesis, an adjusted $p$-value can be obtained by 'trial and error' as the smallest significance level $\alpha$ at which the hypothesis can be rejected by the stepdown multiple testing procedure. But clearly this way of computing adjusted $p$-values would be rather cumbersome. Instead, it is desirable to have an efficient (or streamlined) algorithm for computing adjusted $p$-values. This paper details such an algorithm.

Of course, algorithms for computing $p$-values adjusted for multiple testing have been described before; for example, see Westfall and Young (1993) and the various references to earlier work listed in Section 1.3 of that book. But the contribution of this paper is to describe an algorithm that is custom-tailored to the stepdown multiple testing procedures proposed in Romano and Wolf (2005a,b), which will make it easier for practitioners to understand and implement this algorithm.

\section{Notation and Unadjusted $p$-Values}

We now give a stylized, high-level description of the multiple testing problem under study. The details - such as the construction of test statistics and sufficient conditions for (asymptotic) validity of the proposed stepdown procedures - depend on the context; see Romano and Wolf (2005a,b) and Romano et al. (2008, Section 3).

There are $S$ individual hypothesis testing problems:

$$
H_{s} \quad \text { vs. } \quad H_{s}^{\prime} \quad \text { for } s=1, \ldots, S \text {, }
$$

where $H_{s}$ denotes a null hypothesis and $H_{s}^{\prime}$ denotes an alternative hypothesis. The corresponding test statistics are denoted by $t_{1}, \ldots, t_{S}$. They are designed in a way such that large values are

\footnotetext{
${ }^{1}$ Such adjusted $p$-values are sometimes also called multiplicity-adjusted $p$-values.
} 
indicative of the alternative. (In particular, for two-sided testing problems, the test statistics would usually be based on absolute values.)

Stepdown multiple testing procedures are generally based on a set of null resampling test statistics $\boldsymbol{t}^{*, m}:=\left(t_{1}^{*, m}, \ldots t_{S}^{*, m}\right)$, for $m=1, \ldots, M$, where $M$ denotes the number of resampling repetitions. Depending on context, the resampling can be carried out by a bootstrap method, a permutation method, or a randomization method. Details for the bootstrap method can be found in Romano and Wolf (2005a, Section 4.2), Romano and Wolf (2005b), and Romano et al. (2008, Section 4.3). Details for the permutation and randomization methods can be found in Romano and Wolf (2005a, Section 3.2).

Following Davison and Hinkley (1997, Chapter 4), an unadjusted (or marginal) $p$-value for $H_{s}$, denoted by $\hat{p}_{s}$, can be defined as

$$
\hat{p}_{s}:=\frac{\#\left\{t_{s}^{*, m} \geqslant t_{s}\right\}+1}{M+1} .
$$

Note that this definition of unadjusted $p$-values is not unique. For example, some people instead use the definition

$$
\hat{p}_{s}:=\frac{\#\left\{t_{s}^{*, m} \geqslant t_{s}\right\}}{M} .
$$

Clearly, when $M$ is reasonably large (such as $M=1,000$ ), the difference between (2.1) and (2.2) is not practically relevant.

\section{Stepdown Multiple Testing at Fixed Significance Level}

It will be convenient to first describe the generic stepdown multiple testing procedure that controls the FWE at fixed significance level $\alpha$ in the stylized notation of this paper. In this way, the algorithm to compute the adjusted $p$-values in the next section will be easier to understand.

The hypotheses are relabeled in descending order of the observed test statistics. More specifically, let $\left\{r_{1}, r_{2}, \ldots, r_{S}\right\}$ denote a permutation of $\{1,2, \ldots, S\}$ that satisfies $t_{r_{1}} \geqslant t_{r_{2}} \geqslant \cdots \geqslant t_{r_{S}}$. In this way, $H_{r_{1}}$ is the 'most significant' hypothesis and $H_{r_{S}}$ is the 'least significant' hypothesis.

Let $\max _{t, j}^{*, m}$ denote the largest value of the vector $\left(t_{r_{j}}^{*, m}, \ldots, t_{r_{S}}^{*, m}\right)$, that is,

$$
\max _{t, j}^{*, m}:=\max \left\{t_{r_{j}}^{*, m}, \ldots, t_{r_{S}}^{*, m}\right\} \quad \text { for } j=1, \ldots, S \text { and } m=1, \ldots, M .
$$

Furthermore, let $\hat{c}(1-\alpha, j)$ denote an empirical $1-\alpha$ quantile of the collection $\left\{\max _{t, j}^{*, m}\right\}_{m=1}^{M}$. (There is no unique definition of an empirical quantile. ${ }^{2}$ But as long as $M$ is reasonably large, the differences are not practically relevant.)

The algorithm for the stepdown multiple testing procedure at significance level $\alpha$ is as follows.

\footnotetext{
${ }^{2}$ For example, the statistical software $\mathrm{R}$ offers nine different versions of empirical quantiles in its function quantile. Our recommendation would be to simply use the default version.
} 
Algorithm 3.1 (Stepdown Multiple Testing at Significance Level $\alpha$ ).

1. For $s=1, \ldots, S$, reject $H_{r_{s}}$ iff $t_{r_{s}}>\hat{c}(1-\alpha, 1)$.

2. Denote by $R_{1}$ the number of hypotheses rejected. If $R_{1}=0$, stop; otherwise let $j=2$.

3. For $s=R_{j-1}+1, \ldots, S$, reject $H_{r_{s}}$ iff $t_{r_{s}}>\hat{c}\left(1-\alpha, R_{j-1}+1\right)$.

4. (a) If no further hypotheses are rejected, stop.

(b) Otherwise, denote by $R_{j}$ the number of all hypotheses rejected so far and, afterwards, let $j:=j+1$. Then return to step 3 .

Remark 3.1 (Alternative Description).

It is easy to see that $H_{r_{s}}$ will be rejected at level $\alpha$ by Algorithm 3.1 if and only if

$$
t_{r_{j}}>\hat{c}(1-\alpha, j) \text { for all } j=1, \ldots, s
$$

Therefore, the set of hypotheses rejected at level $\alpha$ is given by the collection $\left\{H_{r_{1}}, \ldots, H_{r_{n}}\right\}$, where $n$ is the largest integer in the set $\{1, \ldots, S\}$ such that $t_{r_{j}}>\hat{c}(1-\alpha, j)$ for all $j=1, \ldots, n$. If no such $n$ exists, then no hypothesis is rejected.

\section{Adjusting $p$-Values for Stepdown Multiple Testing}

We denote the adjusted $p$-value for hypothesis $H_{s}$ by $\hat{p}_{s}^{\text {adj }}$. The following algorithm describes how these adjusted $p$-values can be computed in an efficient manner.

Algorithm 4.1 (Computation of $p$-Values Adjusted for Stepdown Multiple Testing).

1. Define

$$
\hat{p}_{r_{1}}^{\text {adj }}:=\frac{\#\left\{\max _{t, 1}^{*, m} \geqslant t_{r_{1}}\right\}+1}{M+1} .
$$

2. For $s=2, \ldots, S$,

(a) first let

$$
\hat{p}_{r_{s}}^{\text {initial }}:=\frac{\#\left\{\max _{t, s}^{*, m} \geqslant t_{r_{s}}\right\}+1}{M+1},
$$

(b) then enforce monotonicity by defining

$$
\hat{p}_{r_{s}}^{\text {adj }}:=\max \left\{\hat{p}_{r_{s}}^{\text {initial }}, \hat{p}_{r_{s-1}}^{\text {adj }}\right\}
$$


Remark 4.1 (Enforcing Monotonicity).

Step 2.(b) in Algorithm 4.1 is essential. Without it, the adjusted $p$-values for the hypotheses $H_{r_{2}}, \ldots, H_{r_{S}}$ would generally be too optimistic (in the sense of providing evidence against the null). This fact is easiest to see by considering $H_{r_{S}}$. Without step 2.(b), it would hold that $\hat{p}_{r_{S}}^{\text {adj }}=\hat{p}_{r_{S}}$, so that the adjusted $p$-value would be equal to the unadjusted $p$-value.

It is straightforward to see that the adjusted $p$-values are correct in the sense that, as long as $M$ is reasonably large, $H_{s}$ will be rejected at fixed level $\alpha$ by Algorithm 3.1 for all practical purposes if and only if the adjusted $p$-value for $H_{s}$ computed by Algorithm 4.1 satisfies $\hat{p}_{s}^{\text {adj }} \leqslant \alpha$. The addition of "for all practical purposes" to this statement is due to the fact that, as previously mentioned, there exists a unique definition neither for the empirical quantiles $\hat{c}(1-\alpha, j)$ used in Algorithm 3.1 nor for the resampling-based $p$-values used in Algorithm 4.1. But as long as $M$ is reasonably large (such as $M=1,000$ ), violations of the if-and-only-if statement could not occur before the third decimal place of $\alpha$, which is not practically relevant. 


\section{References}

Campbell, F., Conti, G., Heckman, J. J., Moon, S. H., Pinto, R., Pungello, E., and Pan, Y. (2014). Early childhood investmens substantially boost adult health. Science, 343:1478-1485.

Davison, A. C. and Hinkley, D. V. (1997). Bootstrap Methods and their Application. Cambridge University Press, Cambridge.

Dobbie, W. S. and Fryer, R. G. (2015). The medium-term impacts of high-achieving charter schools. Journal of Political Economy, 123(5):985-1037.

Gertler, P., Heckman, J. J., Pinto, R., Zanolini, A., Vermeersch, C., Walker, S., Chang, S. M., and S. Grantham-McGregor (2014). Labor market returns to an early childhood stimulation intervention in Jamaica. Science, 344:998-1001.

Heckman, J. J., Moon, S. H., Pinto, R., Savelyev, P., and Yavitz, A. (2010). Analyzing social experiments as implemented: A reexamination of the evidence from the HighScope Perry Preschool Program. Quantitative Economics, 1(1):1-46.

Hein, G., Silani, G., Preuschoff, K., Batson, C. D., and Singer, T. (2010). Neural responses to ingroup and outgroup members' suffering predict individual differences in costly helping. Neuron, 68:149-160.

Romano, J. P., Shaikh, A. M., and Wolf, M. (2008). Formalized data snooping based on generalized error rates. Econometric Theory, 24(2):404-447.

Romano, J. P. and Wolf, M. (2005a). Exact and approximate stepdown methods for multiple hypothesis testing. Journal of the American Statistical Association, 100(469):94-108.

Romano, J. P. and Wolf, M. (2005b). Stepwise multiple testing as formalized data snooping. Econometrica, 73(4):1237-1282.

Westfall, P. H. and Young, S. S. (1993). Resampling-Based Multiple Testing: Examples and Methods for $p$-Value Adjustment. John Wiley, New York. 